\title{
A FINITENESS RESULT FOR ASSOCIATED PRIMES OF CERTAIN EXT-MODULES
}

\author{
MARKUS BRODMANN and LE THANH NHAN
}

Abstract ${ }^{1}$. Using some properties of unconditioned $M$-sequences in dimension $>s$, we give a finiteness result for the set $\bigcup_{n \in \mathbb{N}} \operatorname{Ass}_{R}\left(\operatorname{Ext}_{R}^{i}\left(R / I^{n}, M\right)\right)$.

\section{Introduction}

Throughout this paper, let $R$ be a Noetherian commutative ring, let $M$ be a finitely generated $R$-module, and $A$ an Artinian $R$-module.

For an ideal $I$ of $R$, it was shown in $[\mathrm{B}]$ that the two sequences of associated primes

$$
\operatorname{Ass}_{R}\left(M / I^{n} M\right) \text { and } \operatorname{Ass}_{R}\left(I^{n} M / I^{n+1} M\right), n=1,2, \ldots
$$

eventually become constant for large $n$. Sharp [Sh] proved the dual result for Artinian modules: $\operatorname{Att}_{R}\left(0:_{A} I^{n}\right)$ and $\operatorname{Att}_{R}\left(\left(0:_{A} I^{n}\right) /\left(0:_{A} I^{n-1}\right)\right)$ do not depend on $n$ for $n$ large. Starting from the observation that $M / I^{n} M \cong \operatorname{Tor}_{0}^{R}\left(R / I^{n}, M\right)$ and $0:_{A} I^{n} \cong \operatorname{Ext}_{R}^{0}\left(R / I^{n}, A\right)$ for any $n$, Melkersson and Schenzel [MS] extended the above results as follows: For any given integer $i \geq 0$, the sequences

$$
\operatorname{Ass}_{R}\left(\operatorname{Tor}_{i}^{R}\left(R / I^{n}, M\right)\right) \text { and } \operatorname{Att}_{R}\left(\operatorname{Ext}_{R}^{i}\left(R / I^{n}, A\right)\right), n=1,2, \ldots
$$

become independent of $n$ for large $n$. Melkersson and Schenzel [MS] also asked whether the set $\operatorname{Ass}_{R}\left(\operatorname{Ext}_{R}^{i}\left(R / I^{n}, M\right)\right)$ is independent of $n$ for large $n$.

In fact, $\bigcup_{n \in \mathbb{N}} \operatorname{Ass}_{R}\left(\operatorname{Ext}_{R}^{i}\left(R / I^{n}, M\right)\right)$ is not a finite set in general, and therefore the set $\operatorname{Ass}_{R}\left(\operatorname{Ext}_{R}^{i}\left(R / I^{n}, M\right)\right)$ depends on $n$ for $n$ large. Indeed, Katzman [Ka, Corollary 1.3] gave an example of a Noetherian local ring $(R, \mathfrak{m})$ with two elements $x, y \in \mathfrak{m}$ such that $\operatorname{Ass}_{R}\left(H_{(x, y) R}^{2}(R)\right)$ is an infinite set. Therefore the set $\bigcup_{n \in \mathbb{N}} \operatorname{Ass}_{R}\left(\operatorname{Ext}_{R}^{2}\left(R /(x, y)^{n}, R\right)\right)$ is infinite.

For convenience, for a subset $T$ of Spec $R$ and an integer $i \geq 0$, we set

$$
\begin{aligned}
(T)_{i}: & =\{\mathfrak{p} \in T: \operatorname{dim} R / \mathfrak{p}=i\} \\
(T)_{\geq i}: & =\{\mathfrak{p} \in T: \operatorname{dim} R / \mathfrak{p} \geq i\}
\end{aligned}
$$

\footnotetext{
${ }^{1}$ Key words and phrases: Supports of local cohomology modules, associated primes, filter regular sequences, $M-$ sequences in dimension $>s$.

2000 Subject Classification: 13D45, 13E05.

The second author was partially supported by the Swiss National Science Foundation (Project No 20-103491/1).
} 
For an integer $i \geq 0$, an ideal $I$ of $R$, and a system $\underline{a}=\left(a_{1}, \ldots, a_{k}\right)$ of elements in $R$, we set

$$
\begin{aligned}
& T^{i}(I, M):=\bigcup_{n \in \mathbb{N}} \operatorname{Ass}_{R}\left(\operatorname{Ext}_{R}^{i}\left(R / I^{n}, M\right)\right) \\
& T^{i}(\underline{a}, M):=\bigcup_{n_{1}, \ldots, n_{k} \in \mathbb{N}} \operatorname{Ass}_{R}\left(\operatorname{Ext}_{R}^{i}\left(R /\left(a_{1}^{n_{1}}, \ldots, a_{k}^{n_{k}}\right), M\right)\right) .
\end{aligned}
$$

In this paper, we prove the following finiteness result for the sets $T^{i}(I, M)$ and $T^{i}(\underline{a}, M)$.

Theorem 1.1. Let $s \geq 0$ and $r \geq 1$ be integers. Assume that $\operatorname{dim}\left(\operatorname{Supp}\left(H_{I}^{i}(M)\right)\right) \leq s$ for all $i<r$. Then for any system of generators $\underline{a}=\left(a_{1}, \ldots, a_{k}\right)$ of $I$ and for all integers $t \leq r$, the

sets $\left(T^{t}(I, M)\right)_{\geq s}$ and $\left(T^{t}(\underline{a}, M)\right)_{\geq s}$ are contained in the finite set $\bigcup_{i=0}^{t} \operatorname{Ass}_{R} \operatorname{Ext}_{R}^{i}(R / I, M)$.

Theorem 1.2. Let $s \geq 0$ and $r \geq 1$ be integers. Assume that $\operatorname{dim}\left(\operatorname{Supp}\left(H_{I}^{i}(M)\right)\right) \leq s$ for all $i<r$. Let $x_{1}, \ldots, x_{r} \in I$ be a sequence which is at the same time an unconditioned $M$-sequence in dimension $>s$ and an unconditioned $I$-filter regular sequence with respect to $M$ (such sequences exist by Proposition 2.5). Then for any system of generators $\underline{a}=$ $\left(a_{1}, \ldots, a_{k}\right)$ of $I$ and for all integers $t \leq r$, the sets $\left(T^{t}(I, M)\right)_{\geq s}$ and $\left(T^{t}(\underline{a}, M)\right)_{\geq s}$ are contained in the finite set

$$
\left(\operatorname{Ass}_{R}\left(M /\left(x_{1}, \ldots, x_{t}\right) M\right)\right)_{\geq s+1} \cup\left(\bigcup_{i=0}^{t} \operatorname{Ass}_{R}\left(M /\left(x_{1}, \ldots, x_{i}\right) M\right)\right)_{s} .
$$

\section{Unconditioned $M$-sequences in dimension $>s$}

Definition 2.1. Let $s \geq 0$ be an integer, let $x_{1}, \ldots, x_{r} \in R$ be a sequence. We say that $x_{1}, \ldots, x_{r}$ is an $M$-sequence in dimension $>s$ if $x_{1}, \ldots, x_{r}$ is a poor $M_{\mathfrak{p}}$-sequence for all $\mathfrak{p} \in \operatorname{Spec}(R)$ with $\operatorname{dim}(R / \mathfrak{p})>s$.

Observe that $x_{1}, \ldots, x_{r}$ is an $M$-sequence in dimension $>s$ if and only if $x_{i} \notin \mathfrak{p}$ for all $\mathfrak{p} \in\left(\operatorname{Ass}_{R}\left(M /\left(x_{1}, \ldots, x_{i-1}\right) M\right)\right)_{\geq s+1}$ and all $i=1, \ldots, r$.

Assume that $R$ is local. Then $x_{1}, \ldots, x_{r}$ is an $M$-sequence in dimension $>0$ if and only if it is a filter regular sequence with respect to $M$ in sense of [Cst]. Moreover, $x_{1}, \ldots, x_{r}$ is an $M$-sequence in dimension $>1$ if and only if it is a generalized regular sequence with respect to $M$ in sense of $[\mathrm{Nh}]$.

Reminder 2.2. (a) Let $I$ be an ideal. A sequence $x_{1}, \ldots, x_{r} \in I$ is called an $I$-filter regular sequence with respect to $M$ if $x_{1}, \ldots, x_{r}$ is an $M_{\mathfrak{p}}$-sequence for all $\mathfrak{p} \in \operatorname{Spec}(R) \backslash \operatorname{Var}(I)$. It is equivalent to say that $x_{i} \notin \mathfrak{p}$ for all $\mathfrak{p} \in \operatorname{Ass}_{R}\left(M /\left(x_{1}, \ldots, x_{i-1}\right) M\right) \backslash \operatorname{Var}(I)$ and all $i=1, \ldots, r$. 
(b) (cf. [NS, 3.4], [Kh, 2.1]). If $x_{1}, \ldots, x_{r}$ is an $I$-filter regular sequence with respect to $M$ then

$$
H_{I}^{j}(M)= \begin{cases}H_{\left(x_{1}, \ldots, x_{r}\right) R}^{j}(M), & \text { if } j<r \\ H_{I}^{j-r}\left(H_{\left(x_{1}, \ldots, x_{r}\right) R}^{r}(M)\right), & \text { if } j \geq r .\end{cases}
$$

Definition 2.3. A sequence $x_{1}, \ldots, x_{r} \in R$ is called an unconditioned $M$-sequence in $d i$ mension $>s$ if $x_{\sigma(1)}, \ldots, x_{\sigma(r)}$ is an $M$-sequence in dimension $>s$ for all permutations $\sigma \in \mathbb{S}_{r}$. Similarly, a sequence $x_{1}, \ldots, x_{r} \in I$ is called an unconditioned $I$-filter regular sequence with respect to $M$ if $x_{\sigma(1)}, \ldots, x_{\sigma(r)}$ is an $I$-filter regular sequence with respect to $M$ for all permutations $\sigma \in \mathbb{S}_{r}$.

Lemma 2.4. Let $s \geq 0$ be an integer, let $I$ be an ideal of $R$.

(a) Let $r>0$ be an integer. Then $\operatorname{dim}\left(\operatorname{Supp}\left(H_{I}^{i}(M)\right)\right) \leq s$ for all $i<r$ if and only if there exists an $M$-sequence in dimension $>s$ of length $r$ in $I$.

(b) If $\operatorname{dim}(M / I M)>s$ then each $M$-sequence in dimension $>s$ in I may be extended to a maximal $M$-sequence in dimension $>s$ in $I$. Moreover, all maximal $M$-sequences in dimension $>s$ in I have the same length, and this common length is equal to the least integer $i$ such that $\operatorname{dim}\left(\operatorname{Supp}\left(H_{I}^{i}(M)\right)\right)>s$.

(c) If $\operatorname{dim}(M / I M) \leq s$ then there exists an $M$-sequence in dimension $>s$ in I of length $n$ for any integer $n>0$.

Proof. (a). Assume that $\operatorname{dim}\left(\operatorname{Supp}\left(H_{I}^{i}(M)\right)\right) \leq s$ for all $i<r$. We prove by induction on $r$ that there is a sequence $x_{1}, \ldots, x_{r} \in I$ which is an $M$-sequence in dimension $>s$. Let $r \geq 1$. Then $\operatorname{dim}\left(\operatorname{Supp}\left(H_{I}^{0}(M)\right)\right) \leq s$. Hence $I \nsubseteq \mathfrak{p}$ for all $\mathfrak{p} \in\left(\operatorname{Ass}_{R} M\right)_{>s+1}$. Therefore there exists an element $x_{1} \in I$ which is $M$-regular in dimension $>s$. This proves the case $r=1$. Let $r>1$ and set $x_{1}=x$. Then $\operatorname{dim}\left(0:_{M} x\right) \leq s$. From the exact sequence $0 \longrightarrow 0:_{M} x \longrightarrow M \longrightarrow M /\left(0:_{M} x\right) \longrightarrow 0$ we get an exact sequence

$$
H_{I}^{i}(M) \longrightarrow H_{I}^{i}\left(M /\left(0:_{M} x\right)\right) \longrightarrow H_{I}^{i+1}\left(0:_{M} x\right)
$$

for all $i \geq 0$. As $\operatorname{dim}\left(0:_{M} x\right) \leq s$, we have $\operatorname{dim}\left(\operatorname{Supp}\left(H_{I}^{i}\left(0:_{M} x\right)\right)\right) \leq s$ for all $i \geq 0$. Therefore, by our hypothesis, $\operatorname{dim}\left(\operatorname{Supp}\left(H_{I}^{i}\left(M /\left(0:_{M} x\right)\right)\right) \leq s\right.$ for all $i<r$. From the exact sequence

$$
0 \longrightarrow M /(0: M x) \longrightarrow M \longrightarrow M / x M \longrightarrow 0
$$

we get an exact sequence $H_{I}^{i}(M) \longrightarrow H_{I}^{i}(M / x M) \longrightarrow H_{I}^{i+1}\left(M /\left(0:_{M} x\right)\right)$ for all $i \geq 0$. So, $\operatorname{dim}\left(\operatorname{Supp}\left(H_{I}^{i}(M / x M)\right)\right) \leq s$ for all $i<r-1$. By induction, there exists a sequence $x_{2}, \ldots, x_{r}$ in $I$ which is an $M / x M$-sequence in dimension $>s$. So, $x_{1}, \ldots, x_{r}$ is an $M$-sequence in dimension $>s$ in $I$.

Conversely, assume $x_{1}, \ldots, x_{r}$ is an $M$-sequence in dimension $>s$ in $I$. Let $\mathfrak{p} \in \operatorname{Spec} R$ such that $\operatorname{dim}(R / \mathfrak{p})>s$. Then $\frac{x_{1}}{1}, \ldots, \frac{x_{r}}{1}$ is a poor $M_{\mathfrak{p}}$-sequence in $I_{\mathfrak{p}}$. So, $H_{I R_{\mathfrak{p}}}^{i}\left(M_{\mathfrak{p}}\right)=0$, i.e. $\mathfrak{p} \notin \operatorname{Supp}\left(H_{I}^{i}(M)\right)$ for all $i<r$. Therefore $\left.\operatorname{dim}\left(\operatorname{Supp} H_{I}^{i}(M)\right)\right) \leq s$ for all $i<r$.

(b). Since $\operatorname{dim}(M / I M)>s$, there is a maximal ideal $\mathfrak{m}$ such that $\operatorname{dim}\left(M_{\mathfrak{m}} / I M_{\mathfrak{m}}\right)>s$. Note that each $M$-sequence in dimension $>s$ in $I$ is an $M_{\mathfrak{m}}$-sequence in dimension $>s$ in $I R_{\mathfrak{m}}$. 
As $\operatorname{dim}\left(M_{\mathfrak{m}} / I M_{\mathfrak{m}}\right)>s$, each $M_{\mathfrak{m}}$-sequence in dimension $>s$ in $I R_{\mathfrak{m}}$ is a part of a system of parameters of $M_{\mathfrak{m}}$. Therefore the length of an $M$-sequence in dimension $>s$ in $I$ is at most $\operatorname{dim} M_{\mathfrak{m}}-s-1$. So, there is a common bound on the lengths of all $M$-sequences in dimension $>s$ which consist of elements in $I$. Therefore, each $M$-sequence in dimension $>s$ in $I$ may be extended to a maximal $M$-sequence in dimension $>s$ in $I$.

Let $x_{1}, \ldots, x_{r}$ and $y_{1}, \ldots, y_{t}$ be maximal $M$-sequences in dimension $>s$ in $I$. Assume that $r \neq t$, say $r<t$. By (a), $\left.\operatorname{dim}\left(\operatorname{Supp} H_{I}^{i}(M)\right)\right) \leq s$ for all $i \leq r$. Similar as in the proof of (a), it follows by induction on $k$ that $\operatorname{dim}\left(\operatorname{Supp}\left(H_{I}^{i}\left(M /\left(x_{1}, \ldots, x_{k}\right) M\right)\right)\right) \leq s$ for all $i \leq r-k$ and all $k \leq r$. Thus $\operatorname{dim}\left(H_{I}^{0}\left(M /\left(x_{1}, \ldots, x_{r}\right) M\right)\right) \leq s$. Therefore there is an element in $I$ which is $M /\left(x_{1}, \ldots, x_{r}\right) M$-regular in dimension $>s$. This is a contradiction to the maximality of the sequence $\left(x_{1}, \ldots, x_{r}\right)$. So, all maximal $M$-sequences in dimension $>s$ in $I$ have the same length and by (a) this length has the stated value.

(c) is clear.

Proposition 2.5. Let $s \geq 0$ and $r \geq 1$ be integers, and let $I \subseteq R$ be an ideal. If $\operatorname{dim}\left(\operatorname{Supp}\left(H_{I}^{i}(M)\right)\right) \leq s$ for all $i<r$ then there is a sequence $x_{1}, \ldots, x_{r}$ in $I$ which is at the same time an unconditioned $M$-sequence in dimension $>s$ and an unconditioned $I$ - filter regular sequence with respect to $M$.

Proof. We proceed by induction on $r$. Let $r=1$. Set

$$
C_{1}:=\left(\operatorname{Ass}_{R} M\right)_{\geq s+1} \cup\left(\operatorname{Ass}_{R} M \backslash \operatorname{Var}(I)\right) .
$$

Since $\operatorname{dim}\left(H_{I}^{0}(M)\right) \leq s$, it follows that $I \nsubseteq \mathfrak{p}$ for all $\mathfrak{p} \in\left(\operatorname{Ass}_{R} M\right)_{\geq s+1}$. Therefore, by Prime Avoidance, there exists an element $x_{1} \in I$ such that $x_{1} \notin \mathfrak{p}$ for all $\mathfrak{p} \in C_{1}$. It is clear that $x_{1}$ is an unconditioned $M$-sequence in dimension $>s$ and an unconditioned $I$-filter regular sequence w.r.t. $M$.

Let $r>1$ and assume that the result is true for $r-1$. Then there exists a sequence $x_{1}, \ldots, x_{r-1}$ in $I$ which is an unconditioned $M$-sequence in dimension $>s$ and an unconditioned $I$-filter regular sequence w.r.t. $M$. By Lemma 2.4 and by the assumption, for any subset $J$ of $\{1, \ldots, r-1\}$, the sequence $\left(x_{j}\right)_{j \in J}$ can be extended to an $M$-sequence in dimension $>s$ in $I$ of length $r$. Therefore for any subset $J$ of $\{1, \ldots, r-1\}$, there exists an $\left(M / \sum_{j \in J} x_{j} M\right)$-regular element in dimension $>s$ in $I$. It follows that $I \nsubseteq \mathfrak{p}$ for all $\mathfrak{p} \in\left(\operatorname{Ass}_{R}\left(M / \sum_{j \in J} x_{j} M\right)\right)_{\geq s+1}$ and all subsets $J$ of $\{1, \ldots, r-1\}$. By Prime Avoidance, we can choose an element $x_{r} \in I$ such that $x_{r} \notin \mathfrak{p}$ for all $\mathfrak{p} \in C_{r}$, where

$$
C_{r}:=\left(\bigcup_{J \subseteq\{1, \ldots, r-1\}} \operatorname{Ass}_{R}\left(M / \sum_{j \in J} x_{j} M\right)\right)_{\geq s+1} \cup\left(\bigcup_{J \subseteq\{1, \ldots, r-1\}} \operatorname{Ass}_{R}\left(M / \sum_{j \in J} x_{j} M\right) \backslash \operatorname{Var}(I)\right) .
$$

We first show that $x_{1}, \ldots, x_{r}$ is an unconditioned $M$-sequence in dimension $>s$. Let $\sigma \in \mathbb{S}_{r}$ be a permutation of $1, \ldots, r$. Assume that $x_{\sigma(1)}, \ldots, x_{\sigma(r)}$ is not an $M$-sequence in dimension $>s$. Let $n \in\{1, \ldots, r\}$ be the least integer such that $x_{\sigma(1)}, \ldots, x_{\sigma(n)}$ is not an $M$-sequence in dimension $>s$. Then $r=\sigma(i)$ for some $i<n$ by our choice 
of $x_{r}$, and there is some $\mathfrak{p} \in\left(\operatorname{Ass}_{R}\left(M /\left(x_{\sigma(1)}, \ldots, x_{\sigma(n-1)}\right) M\right)\right)_{\geq s+1}$ such that $x_{\sigma(n)} \in \mathfrak{p}$. So $x_{\sigma(1)}, \ldots, x_{\sigma(n)} \in \mathfrak{p}$ and $\frac{x_{\sigma(1)}}{1}, \ldots, \frac{x_{\sigma(n)}}{1}$ is not a regular sequence w.r.t. $M_{\mathfrak{p}}$. Therefore $\frac{x_{\sigma(1)}}{1}, \ldots, \frac{x_{\sigma(i-1)}}{1}, \frac{x_{\sigma(i+1)}}{1}, \ldots, \frac{x_{\sigma(n)}}{1}, \frac{x_{\sigma(i)}}{1}$ is not a regular sequence w.r.t. $M_{\mathfrak{p}}$. Set $J:=\{j \in \mathbb{N}: j \leq n, j \neq i\}$. As $\operatorname{dim}(R / \mathfrak{p})>s$, we know that $\left(\frac{x_{\sigma(j)}}{1}\right)_{j \in J}$ is a regular sequence w.r.t. $M_{\mathfrak{p}}$. Therefore $\frac{x_{r}}{1}=\frac{x_{\sigma(i)}}{1}$ is not a regular element w.r.t. $M_{\mathfrak{p}} / \sum_{j \in J} x_{\sigma(j)} M_{\mathfrak{p}}$. So, there exists $\mathfrak{q} \in \operatorname{Spec}(R)$ with $\mathfrak{q} \subseteq \mathfrak{p}$ such that $\frac{x_{r}}{1} \in \mathfrak{q} R_{\mathfrak{p}} \in \operatorname{Ass}_{R_{\mathfrak{p}}}\left(M_{\mathfrak{p}} / \sum_{j \in J} x_{\sigma(j)} M_{\mathfrak{p}}\right)$. It follows that $x_{r} \in \mathfrak{q} \in\left(\operatorname{Ass}_{R}\left(M / \sum_{j \in J} x_{\sigma(j)} M\right)\right)_{\geq s+1} \subseteq C_{r}$. This gives a contradiction.

Finally, we need to prove that $x_{1}, \ldots, x_{r}$ is an unconditioned $I$-filter regular sequence w.r.t. $M$. Assume that $x_{\sigma(1)}, \ldots, x_{\sigma(r)}$ is not an $I$-filter regular sequence w.r.t. $M$ for some $\sigma \in \mathbb{S}_{r}$. Let $n$ be the least integer such that $x_{\sigma(1)}, \ldots, x_{\sigma(n)}$ is not an $I$-filter regular sequence w.r.t. $M$. By our choice of $x_{r}$, we have $r=\sigma(i)$ for some $i<n$ and there exists some $\mathfrak{p} \in \operatorname{Ass}_{R}\left(M /\left(x_{\sigma(1)}, \ldots, x_{\sigma(n-1)}\right) M\right) \backslash \operatorname{Var}(I)$ such that $x_{\sigma(n)} \in \mathfrak{p}$. So $x_{\sigma(1)}, \ldots, x_{\sigma(n)} \in \mathfrak{p}$ and $\frac{x_{\sigma(1)}}{1}, \ldots, \frac{x_{\sigma(n)}}{1}$ is not a regular sequence w.r.t. $M_{\mathfrak{p}}$. Therefore $\frac{x_{\sigma(1)}}{1}, \ldots, \frac{x_{\sigma(i-1)}}{1}, \frac{x_{\sigma(i+1)}}{1}, \ldots, \frac{x_{\sigma(n)}}{1}, \frac{x_{\sigma(i)}}{1}$ is not a regular sequence w.r.t. $M_{\mathfrak{p}}$. Set $J:=\{j \in \mathbb{N}: j \leq n, j \neq i\}$. As $\mathfrak{p} \notin \operatorname{Var}(I)$, we know that $\left(\frac{x_{\sigma(j)}}{1}\right)_{j \in J}$ is a regular sequence w.r.t. $M_{\mathfrak{p}}$. Therefore $\frac{x_{r}}{1}=\frac{x_{\sigma(i)}}{1}$ is not a regular element w.r.t. $M_{\mathfrak{p}} / \sum_{j \in J} x_{\sigma(j)} M_{\mathfrak{p}}$. So, there exists some $\mathfrak{q} \in \operatorname{Spec}(R)$ with $\mathfrak{q} \subseteq \mathfrak{p}$ such that $\frac{x_{r}}{1} \in \mathfrak{q} R_{\mathfrak{p}} \in \operatorname{Ass}_{R_{\mathfrak{p}}}\left(M_{\mathfrak{p}} / \sum_{j \in J} x_{\sigma(j)} M_{\mathfrak{p}}\right)$. It follows that $x_{r} \in \mathfrak{q} \in\left(\operatorname{Ass}_{R}\left(M / \sum_{j \in J} x_{\sigma(j)} M\right)\right)_{\geq s+1} \backslash \operatorname{Var}(I) \subseteq C_{r}$, a contradiction.

Proposition 2.6. Let $s$ be a non-negative integer. Let $x_{1}, \ldots, x_{t}$ be an unconditioned $M-$ sequence in dimension $>s$. Then

$$
\left.\left(\bigcup_{n_{1}, \ldots, n_{t} \in \mathbb{N}} \operatorname{Ass}_{R}\left(M /\left(x_{1}^{n_{1}}, \ldots, x_{t}^{n_{t}}\right) M\right)\right)_{\geq s}=\left(\operatorname{Ass}_{R}\left(M /\left(x_{1}, \ldots, x_{t}\right) M\right)\right)\right)_{\geq s} .
$$

In particular, the set $\left(\bigcup_{n_{1}, \ldots, n_{t} \in \mathbb{N}} \operatorname{Ass}_{R}\left(M /\left(x_{1}^{n_{1}}, \ldots, x_{t}^{n_{t}}\right) M\right)\right)_{\geq s}$ is finite.

Proof. We prove the result by induction on $t$. Let $t=1$ and we write $x_{1}=x$. We will show by induction on $n_{1}=n$ that $\left(\operatorname{Ass}_{R}\left(M / x^{n} M\right)\right)_{\geq s} \subseteq \operatorname{Ass}_{R}(M / x M)$. The case $n=1$ is clear. 
Let $n>1$ and assume that the result is true for $n-1$. Let $\mathfrak{p} \in\left(\operatorname{Ass}_{R}\left(M / x^{n} M\right)\right)_{\geq s}$. Then $\mathfrak{p} R_{\mathfrak{p}} \in \operatorname{Ass}_{R_{\mathfrak{p}}}\left(M_{\mathfrak{p}} / x^{n} M_{\mathfrak{p}}\right)$. If $\operatorname{dim}(R / \mathfrak{p})>s$ then $x$ is a regular element w.r.t. $M_{\mathfrak{p}}$ and therefore $\mathfrak{p} R_{\mathfrak{p}} \in \operatorname{Ass}_{R_{\mathfrak{p}}}\left(M_{\mathfrak{p}} / x M_{\mathfrak{p}}\right)$. It follows that $\mathfrak{p} \in \operatorname{Ass}_{R}(M / x M)$. Assume that $\operatorname{dim}(R / \mathfrak{p})=s$. From the exact sequence

$$
0 \longrightarrow x^{n-1} M / x^{n} M \longrightarrow M / x^{n} M \longrightarrow M / x^{n-1} M \longrightarrow 0,
$$

we have $\mathfrak{p} \in \operatorname{Ass}_{R}\left(M / x^{n-1} M\right) \cup \operatorname{Ass}_{R}\left(x^{n-1} M / x^{n} M\right)$. If $\mathfrak{p} \in \operatorname{Ass}_{R}\left(M / x^{n-1} M\right)$ then by induction $\mathfrak{p} \in \operatorname{Ass}_{R}(M / x M)$. So, assume that $\mathfrak{p} \in \operatorname{Ass}_{R}\left(x^{n-1} M / x^{n} M\right)$. Consider the exact sequence

$$
0 \longrightarrow\left(x^{n} M:_{M} x^{n-1}\right) / x M \longrightarrow M / x M \longrightarrow x^{n-1} M / x^{n} M \longrightarrow 0 .
$$

Set $K=\left(x^{n} M:_{M} x^{n-1}\right) / x M$. Assume that $\mathfrak{p} \notin \operatorname{Supp}(K)$. Then the above exact sequence shows that $(M / x M)_{\mathfrak{p}} \cong\left(x^{n-1} M / x^{n} M\right)_{\mathfrak{p}}$. Since $\mathfrak{p} \in \operatorname{Ass}_{R}\left(x^{n-1} M / x^{n} M\right)$, it follows that $\mathfrak{p} \in$ $\operatorname{Ass}_{R}(M / x M)$. So, we assume that $\mathfrak{p} \in \operatorname{Supp}(K)$. Note that for any $\mathfrak{q} \in \operatorname{Supp}(M)$ satisfying $\operatorname{dim}(R / \mathfrak{q})>s$, we have $K_{\mathfrak{q}}=0$ as $x$ is a poor regular element w.r.t. $M_{\mathfrak{q}}$. So $\operatorname{dim}(K) \leq s$. Hence $\mathfrak{p}$ is a minimal element in $\operatorname{Supp}(K)$, and hence $\mathfrak{p} \in \operatorname{Ass}_{R}(K) \subseteq \operatorname{Ass}_{R}(M / x M)$. So, the result is true for $t=1$.

Let $t>1$ and assume that the result is true for $t-1$. Let $n_{1}, \ldots, n_{t}$ be arbitrary positive integers. Since $x_{t}$ is an $M /\left(x_{1}^{n_{1}}, \ldots, x_{t-1}^{n_{t-1}}\right) M$-regular element in dimension $>s$, we get by the case $t=1$ that

$$
\left(\operatorname{Ass}_{R}\left(M /\left(x_{1}^{n_{1}}, \ldots, x_{t}^{n_{t}}\right) M\right)\right)_{\geq s} \subseteq \operatorname{Ass}_{R}\left(M /\left(x_{1}^{n_{1}}, \ldots, x_{t-1}^{n_{t-1}}, x_{t}\right) M\right) .
$$

Since $x_{1}, \ldots, x_{t-1}$ is an unconditioned $M / x_{t} M$-sequence in dimension $>s$, we have by induction that

$$
\begin{aligned}
\left(\operatorname{Ass}_{R}\left(M /\left(x_{1}^{n_{1}}, \ldots, x_{t-1}^{n_{t-1}}, x_{t}\right) M\right)\right)_{\geq s} & =\left(\operatorname{Ass}_{R}\left(M /\left(x_{t}, x_{1}^{n_{1}}, \ldots, x_{t-1}^{n_{t-1}}\right) M\right)\right)_{\geq s} \\
& \subseteq \operatorname{Ass}_{R}\left(M /\left(x_{1}, \ldots, x_{t}\right) M\right) .
\end{aligned}
$$

This proves our claim.

In general, a permutation of an $M$-sequence in dimension $>s$ is not an $M$-sequence in dimension $>s$. In particular, a permutation of a generalized regular sequence is not necessarily a generalized regular sequence. For example, let $R=k\left[\left[x_{1}, x_{2}, x_{3}, x_{4}, x_{5}\right]\right]$ be the ring of power series in 5 variables over a field $k$. Let $M=R /\left(x_{1}\right) \cap\left(x_{2}, x_{3}, x_{4}\right)$. Then $\operatorname{dim} M=4$ and $x_{5}, x_{2}$ is a generalized regular sequence w.r.t $M$, but $x_{2}, x_{5}$ is not a generalized regular sequence w.r.t $M$. This fact shows that Lemma $2.2(\mathrm{i})$,(ii) of [Nh] is not correct. It follows that the proof of $[\mathrm{Nh}, 3.1]$ is not correct, too. The next corollary is an improvement and also a correction of [Nh, 3.1].

Corollary 2.7. Assume that $x_{1}, \ldots, x_{t}$ is an unconditioned generalized regular sequence w.r.t. M. Then

$$
\left.\bigcup_{n_{1}, \ldots, n_{t} \in \mathbb{N}} \operatorname{Ass}_{R}\left(M /\left(x_{1}^{n_{1}}, \ldots, x_{t}^{n_{t}}\right) M\right) \backslash \operatorname{Max} R \subseteq \operatorname{Ass}_{R}\left(M /\left(x_{1}, \ldots, x_{t}\right) M\right)\right) .
$$


In particular, if in addition $R$ is local, then $\bigcup_{n_{1}, \ldots, n_{t} \in \mathbb{N}} \operatorname{Ass}_{R}\left(M /\left(x_{1}^{n_{1}}, \ldots, x_{t}^{n_{t}}\right) M\right)$ is a finite set.

Proof. The proof is immediate by setting $s=1$ in Proposition 2.6.

\section{Finiteness results}

Remark 3.1. Let $I \subseteq R$ be an ideal with $\operatorname{depth}(I, M)=t$. Then it is well known that

$$
\operatorname{Ass}_{R}\left(\operatorname{Ext}_{R}^{t}(R / I, M)\right)=\operatorname{Ass}_{R}\left(H_{I}^{t}(M)\right) .
$$

Lemma 3.2. Let $t$ be a positive integer. Set $P_{t}=\bigcup_{i=0}^{t-1} \operatorname{Supp}\left(\operatorname{Ext}_{R}^{i}(R / I, M)\right)$. Then

$$
\begin{aligned}
& \operatorname{Ass}_{R}\left(\operatorname{Ext}_{R}^{t}\left(R / I^{n}, M\right)\right) \cup P_{t}=\operatorname{Ass}_{R}\left(\operatorname{Ext}_{R}^{t}\left(R /\left(a_{1}^{n_{1}}, \ldots, a_{k}^{n_{k}}\right), M\right)\right) \cup P_{t} \\
& =\operatorname{Ass}_{R}\left(\operatorname{Ext}_{R}^{t}(R / I, M)\right) \cup P_{t}=\operatorname{Ass}_{R}\left(H_{I}^{t}(M)\right) \cup P_{t}
\end{aligned}
$$

for any system of generators $\left(a_{1}, \ldots, a_{k}\right)$ of $I$ and all positive integers $n, n_{1}, \ldots, n_{k}$.

Proof. Let $\mathfrak{p} \in \operatorname{Supp}(M)$ such that $\mathfrak{p} \notin P_{t}$. Then for any $i<t$ we have

$$
\operatorname{Ext}_{R_{\mathfrak{p}}}^{i}\left(R_{\mathfrak{p}} / I R_{\mathfrak{p}}, M_{\mathfrak{p}}\right) \cong\left(\operatorname{Ext}_{R}^{i}(R / I, M)\right)_{\mathfrak{p}}=0 .
$$

Therefore $\operatorname{depth}\left(I R_{\mathfrak{p}}, M_{\mathfrak{p}}\right) \geq t$. Let $n, n_{1}, \ldots, n_{k}$ be positive integers. It is clear that

$$
\operatorname{depth}\left(I^{n} R_{\mathfrak{p}}, M_{\mathfrak{p}}\right)=\operatorname{depth}\left(I R_{\mathfrak{p}}, M_{\mathfrak{p}}\right)=\operatorname{depth}\left(\left(a_{1}^{n_{1}}, \ldots, a_{k}^{n_{k}}\right) R_{\mathfrak{p}}, M_{\mathfrak{p}}\right) .
$$

We first suppose that $\operatorname{depth}\left(I R_{\mathfrak{p}}, M_{\mathfrak{p}}\right)>t$. Then the $R_{\mathfrak{p}}-$ modules $\operatorname{Ext}_{R_{\mathfrak{p}}}^{t}\left(R_{\mathfrak{p}} / I^{n} R_{\mathfrak{p}}, M_{\mathfrak{p}}\right)$, $\operatorname{Ext}_{R_{\mathfrak{p}}}^{t}\left(R_{\mathfrak{p}} /\left(a_{1}^{n_{1}}, \ldots, a_{k}^{n_{k}}\right) R_{\mathfrak{p}}, M_{\mathfrak{p}}\right), \operatorname{Ext}_{R_{\mathfrak{p}}}^{t}\left(R_{\mathfrak{p}} / I R_{\mathfrak{p}}, M_{\mathfrak{p}}\right), H_{I R_{\mathfrak{p}}}^{t}\left(M_{\mathfrak{p}}\right)$ are zero. So, $\mathfrak{p}$ does not belong to any of the four sets $\operatorname{Ass}_{R}\left(\operatorname{Ext}_{R}^{t}\left(R / I^{n}, M\right)\right), \operatorname{Ass}_{R}\left(\operatorname{Ext}_{R}^{t}\left(R /\left(a_{1}^{n_{1}}, \ldots, a_{k}^{n_{k}}\right), M\right)\right)$, $\operatorname{Ass}_{R}\left(\operatorname{Ext}_{R}^{t}(R / I, M)\right)$ and $\operatorname{Ass}_{R}\left(H_{I}^{t}(M)\right)$.

Assume that $\operatorname{depth}\left(I R_{\mathfrak{p}}, M_{\mathfrak{p}}\right)=t$. Since $\operatorname{rad}(I)=\operatorname{rad}\left(I^{n}\right)=\operatorname{rad}\left(\left(a_{1}^{n_{1}}, \ldots, a_{k}^{n_{k}}\right) R\right)$, we have by Remark 3.1 that

$$
\begin{aligned}
& \operatorname{Ass}_{R_{\mathfrak{p}}}\left(\operatorname{Ext}_{R_{\mathfrak{p}}}^{t}\left(R_{\mathfrak{p}} / I^{n} R_{\mathfrak{p}}, M_{\mathfrak{p}}\right)\right)=\operatorname{Ass}_{R_{\mathfrak{p}}}\left(H_{I^{n} R_{\mathfrak{p}}}^{t}\left(M_{\mathfrak{p}}\right)\right)=\operatorname{Ass}_{R_{\mathfrak{p}}}\left(H_{I R_{\mathfrak{p}}}^{t}\left(M_{\mathfrak{p}}\right)\right) \\
&=\operatorname{Ass}_{R_{\mathfrak{p}}}\left(\operatorname{Ext}_{R_{\mathfrak{p}}}^{t}\left(R_{\mathfrak{p}} / I R_{\mathfrak{p}}, M_{\mathfrak{p}}\right)\right), \\
& \operatorname{Ass}_{R_{\mathfrak{p}}}\left(\operatorname{Ext}_{R_{\mathfrak{p}}}^{t}\left(R_{\mathfrak{p}} /\left(a_{1}^{n_{1}}, \ldots, a_{k}^{n_{k}}\right) R_{\mathfrak{p}}, M_{\mathfrak{p}}\right)\right)=\operatorname{Ass}_{R_{\mathfrak{p}}}\left(H_{I R_{\mathfrak{p}}}^{t}\left(M_{\mathfrak{p}}\right)\right) .
\end{aligned}
$$

It follows that

$$
\begin{aligned}
\mathfrak{p} \in \operatorname{Ass}_{R}\left(\operatorname{Ext}_{R}^{t}\left(R / I^{n}, M\right)\right. & \Leftrightarrow \mathfrak{p} \in \operatorname{Ass}_{R}\left(H_{I}^{t}(M)\right) \Leftrightarrow \mathfrak{p} \in \operatorname{Ass}_{R}\left(\operatorname{Ext}_{R}^{t}(R / I, M)\right. \\
& \Leftrightarrow \mathfrak{p} \in \operatorname{Ass}_{R}\left(\operatorname{Ext}_{R}^{t}\left(R /\left(a_{1}^{n_{1}}, \ldots, a_{k}^{n_{k}}\right), M\right) .\right.
\end{aligned}
$$

Now the result follows immediately. 
The next lemma follows easily by induction on $t$ using Remark 3.1 and Lemma 3.2.

Lemma 3.3. For any integer $t \geq 0$ we have

$$
\bigcup_{i=0}^{t}\left(\bigcup_{n \in \mathbb{N}} \operatorname{Supp}\left(\operatorname{Ext}_{R}^{i}\left(R / I^{n}, M\right)\right)\right)=\bigcup_{i=0}^{t} \operatorname{Supp}\left(\operatorname{Ext}_{R}^{i}(R / I, M)\right)=\bigcup_{i=0}^{t} \operatorname{Supp}\left(H_{I}^{i}(M)\right) \text {. }
$$

It should be mentioned that the equalities $\operatorname{Ass}_{R}\left(\operatorname{Ext}_{R}^{t}(R / I, M)\right) \cup P_{t}=\operatorname{Ass}_{R}\left(H_{I}^{t}(M)\right) \cup P_{t}$ in Lemma 3.2 and $\bigcup_{i=0}^{t} \operatorname{Supp}\left(\operatorname{Ext}_{R}^{i}(R / I, M)\right)=\bigcup_{i=0}^{t} \operatorname{Supp}\left(H_{I}^{i}(M)\right)$ in Lemma 3.3 have been proved by Cuong-Hoang $[\mathrm{CH}]$.

Proof of Theorem 1.1. Let $t \leq r$ be a non-negative integer. Set $P_{t}=\bigcup_{i=0}^{t-1} \operatorname{Supp}\left(\operatorname{Ext}_{R}^{i}(R / I, M)\right)$. Then $P_{t}=\bigcup_{i=0}^{t-1} \operatorname{Supp}\left(H_{I}^{i}(M)\right)$ by Lemma 3.3. Therefore, by our assumption, $\operatorname{dim}(R / \mathfrak{p}) \leq s$ for all $\mathfrak{p} \in P_{t}$. Now, let $\mathfrak{p} \in\left(T^{t}(I, M)\right)_{\geq s} \cup\left(T^{t}(\underline{a}, M)\right)_{\geq s}$. If $\operatorname{dim}(R / \mathfrak{p})>s$ then $\mathfrak{p} \notin P_{t}$. So, we get by Lemma 3.2 that $\mathfrak{p} \in \operatorname{Ass}_{R}\left(\operatorname{Ext}_{R}^{t}(R / I, M)\right)$.

Let $\operatorname{dim}(R / \mathfrak{p})=s$. It follows by Lemma 3.2 that $\mathfrak{p} \in\left(\operatorname{Ass}\left(\operatorname{Ext}_{R}^{t}(R / I, M) \cup P_{t}\right)_{s}\right.$. If $\mathfrak{p} \notin \operatorname{Ass}_{R}\left(\operatorname{Ext}_{R}^{t}(R / I, M)\right)$ then $\mathfrak{p} \in P_{t}$, and hence $\mathfrak{p}$ is a minimal prime ideal of $\operatorname{Ext}_{R}^{i}(R / I, M)$ for some $i<t$. Therefore $\mathfrak{p} \in \operatorname{Ass}_{R}\left(\operatorname{Ext}_{R}^{i}(R / I, M)\right)$ for some $i<t$. Thus, $T^{t}(I, M)$ and $T^{t}(\underline{a}, M)$ are subsets of $\bigcup_{i=0}^{t} \operatorname{Ass}\left(\operatorname{Ext}_{R}^{i}(R / I, M)\right)$.

Proof of Theorem 1.2. Let $t \leq r$ be a non-negative integer. Let $\mathfrak{p} \in\left(T^{t}(I, M)\right)_{\geq s} \cup$ $\left(T^{t}(\underline{a}, M)\right)_{\geq s}$. Let $i<t$. Since $\operatorname{dim}\left(\operatorname{Supp}\left(H_{I}^{i}(M)\right)\right) \leq s$, any prime ideal in $\left(\operatorname{Supp}\left(H_{I}^{i}(M)\right)\right)_{\geq s}$ is minimal in $\operatorname{Supp}\left(H_{I}^{i}(M)\right)$ and hence associated to $H_{I}^{i}(M)$. It follows that

$$
\left(\operatorname{Supp}\left(H_{I}^{i}(M)\right)\right)_{\geq s}=\left(\operatorname{Ass}_{R}\left(H_{I}^{i}(M)\right)\right)_{s} \text { for all } i<t .
$$

So, we get by Lemma 3.2 and Lemma 3.3 that $\mathfrak{p} \in\left(\bigcup_{i=0}^{t-1} \operatorname{Ass}_{R}\left(H_{I}^{i}(M)\right)\right)_{s} \cup\left(\operatorname{Ass}_{R}\left(H_{I}^{t}(M)\right)\right)_{\geq s}$. As $x_{1}, \ldots, x_{r} \in I$ is an unconditioned $I$-filter regular sequence w.r.t. $M$, we have by 2.2 that $H_{I}^{i}(M) \cong H_{I}^{0}\left(H_{\left(x_{1}, \ldots, x_{i}\right) R}^{i}(M)\right)$ for all $i=1, \ldots, t$. Therefore, for each $i=1, \ldots, t$, we have

$$
\operatorname{Ass}_{R} H_{I}^{i}(M) \subseteq \operatorname{Ass}_{R}\left(H_{\left(x_{1}, \ldots, x_{i}\right) R}^{i}(M)\right) \subseteq \bigcup_{n \in \mathbb{N}} \operatorname{Ass}_{R}\left(M /\left(x_{1}^{n}, \ldots, x_{i}^{n}\right) M\right) .
$$

As $x_{1}, \ldots, x_{r}$ is an unconditioned $M$-sequence in dimension $>s$, we get by Proposition 2.6 that

$$
\left(\bigcup_{n \in N} \operatorname{Ass}_{R}\left(M /\left(x_{1}^{n}, \ldots, x_{i}^{n}\right) M\right)\right)_{s}=\left(\operatorname{Ass}_{R}\left(M /\left(x_{1}, \ldots, x_{i}\right) M\right)\right)_{s}
$$


for all $i=1, \ldots, t$, and

$$
\left(\bigcup_{n \in N} \operatorname{Ass}_{R}\left(M /\left(x_{1}^{n}, \ldots, x_{t}^{n}\right) M\right)\right)_{\geq s+1}=\left(\operatorname{Ass}_{R}\left(M /\left(x_{1}, \ldots, x_{t}\right) M\right)\right)_{\geq s+1} .
$$

If $\operatorname{dim}(R / \mathfrak{p})>s$ then $t=r$ and $\mathfrak{p} \in \operatorname{Ass}_{R} H_{I}^{t}(M)$, hence $\mathfrak{p} \in\left(\operatorname{Ass}_{R}\left(M /\left(x_{1}, \ldots, x_{t}\right) M\right)\right)_{\geq s+1}$. If $\operatorname{dim}(R / \mathfrak{p})=s$ then

$$
\mathfrak{p} \in \bigcup_{i=0}^{t}\left(\operatorname{Ass}_{R}\left(H_{I}^{i}(M)\right)\right)_{s}=\bigcup_{i=0}^{t}\left(\operatorname{Ass}_{R}\left(M /\left(x_{1}, \ldots, x_{i}\right) M\right)\right)_{s} .
$$

Now the result follows immediately.

Replacing $s$ by 1 in Theorems 1,2 and on use of Lemma 3.2 we get the following result.

Corollary 3.4. Let $r>0$ be an integer such that $\operatorname{dim}\left(\operatorname{Supp}\left(H_{I}^{i}(M)\right)\right) \leq 1$ for all $i<r$. Let $\underline{a}=\left(a_{1}, \ldots, a_{k}\right)$ be a system of generators of $I$. Then

(a) For any integer $t \leq r$, the sets $T^{t}(I, M)$ and $T^{t}(\underline{a}, M)$ are contained in the set

$$
\bigcup_{i=0}^{t} \operatorname{Ass}_{R}\left(\operatorname{Ext}_{R}^{i}(R / I, M)\right) \cup\left(\operatorname{Max}(R) \cap \bigcup_{i=0}^{t-1} \operatorname{Supp}\left(H_{I}^{i}(M)\right)\right) \text {. }
$$

(b) Let $x_{1}, \ldots, x_{r} \in I$ be an unconditioned generalized regular sequence with respect to $M$ which is an unconditioned $I$-filter regular sequence with respect to $M$ (such sequences exist by Proposition 2.5). Then for any integer $t \leq r$, the sets $T^{t}(I, M)$ and $T^{t}(\underline{a}, M)$ are contained in the set $\left(\operatorname{Ass}_{R}\left(M /\left(x_{1}, \ldots, x_{t}\right) M\right)\right)_{\geq 2} \cup\left(\bigcup_{i=0}^{t} \operatorname{Ass}_{R}\left(M /\left(x_{1}, \ldots, x_{i}\right) M\right)\right)_{1} \cup \operatorname{Max}(R)$.

Remark 3.5. Let $R$ be local. In Khashyarmanesh [Kh], it is shown that if $H_{I}^{i}(M)$ is of finite support for all $i<r$ then the set $\left(T^{r}(I, M)\right)_{\geq 2}=\left\{\mathfrak{p} \in T^{r}(I, M): \operatorname{dim} R / \mathfrak{p}>1\right\}$ is finite. Corollary 3.4 shows that even $T^{r}(I, M)$ is a finite set.

Setting $s=0$ in Theorems 1,2 we get the following result.

Corollary 3.6. Let $r>0$ be an integer such that $\operatorname{dim}\left(\operatorname{Supp}\left(H_{I}^{i}(M)\right)\right) \leq 0$ for all $i<r$. Let $\underline{a}=\left(a_{1}, \ldots, a_{k}\right)$ be a system of generators of $I$. Then

(a) For any integer $t \leq r$, the sets $T^{t}(I, M)$ and $T^{t}(\underline{a}, M)$ are contained in the finite set $\bigcup_{i=0}^{t} \operatorname{Ass}_{R}\left(\operatorname{Ext}_{R}^{i}(R / I, M)\right)$.

(b) Let $x_{1}, \ldots, x_{r} \in I$ be an unconditioned filter regular sequence with respect to $M$ which is an unconditioned $I$-filter regular sequence with respect to $M$ (such sequences exist by Proposition 2.5). Then for any integer $t \leq r$, the sets $T^{t}(I, M)$ and $T^{t}(\underline{a}, M)$ are contained in the finite $\operatorname{set}\left(\operatorname{Ass}_{R}\left(M /\left(x_{1}, \ldots, x_{t}\right) M\right)\right)_{\geq 1} \cup \bigcup_{i=0}^{t} \operatorname{Ass}_{R}\left(M /\left(x_{1}, \ldots, x_{i}\right) M\right)$. 
Acknowledgment. The second author is deeply grateful to the Institute of Mathematics of the University of Zürich (Switzerland) for the support while completing this work.

\section{References}

[M] M. Brodmann, Asymptotic stability of $\operatorname{Ass}_{R}\left(M / I^{n} M\right)$, Proc. Amer. Math. Soc., (1) 74 (1979), 16-18.

[BS] M. Brodmann and R. Y. Sharp, "Local cohomology: an algebraic introduction with geometric applications", Cambridge University Press, 1998.

$[\mathrm{CH}]$ N. T. Cuong and N. V. Hoang, Some finite properties of generalized local cohomology modules, Proceeding of the second Japan-Vietnam joint seminar on Commutative Algebra, March 20-26, 2006, Meiji University.

[Cts] N. T. Cuong, P. Schenzel and N. V. Trung, Verallgemeinerte Cohen-Macaulay Moduln, Math. Nachr, 85 (1978), 57-73.

[Ka] M. Katzman, An example of an infinite set of associated primes of a local cohomology module, J. Algebra, 252 (2002), 161-166.

[Kh] K. Khashyarmanesh, On the finiteness properties of $\bigcup_{i} \operatorname{Ass}_{R}\left(\operatorname{Ext}_{R}^{i}\left(R / \mathfrak{a}^{i}, M\right)\right)$, Comm. Algebra, 34 (2006), 779-784.

[NS] U. Nagel and P. Schenzel, Cohomological annihilators and Castelnuovo-Mumford regularity, In: Commutative Algebra: Syzygies, Multiplicities, and Birational Algebra (South Hadley, 1992). Contemp. Math., 159. Amer. Math. Soc., (1994), 307-328.

[Nh] L. T. Nhan, On generalized regular sequences and the finiteness for associated primes of local cohomology modules, Comm. Algebra, 33 (2005), 793-806.

[MS] L. Melkersson and P. Schenzel, Asymptotic prime ideals related to derived functors, Proc. Amer. Math. Soc., (4) 117 (1993), 935-938.

[Sh] R. Y. Sharp, Asymptotic behaviour of certain sets of attached prime ideals, J. London Math. Soc., (2) 34 (1986), 212-218.

Institute of Mathematics, University of Zürich, Zürich, Switzerland

E-mail address: brodmann@math.unizh.ch

Natural Sciences College, Thai Nguyen University, Thai Nguyen, Vietnam

E-mail address: trtrnhan@yahoo.com 